Revista Médica Sinergia

Vol.3 Num: 10

Octubre 2018 pp: 3 - 6

ISSN:2215-4523

e-ISSN:2215-5279

http://revistamedicasinergia.com

\section{ACALASIA ESOFÁGICA}

(Esophageal achalasia)

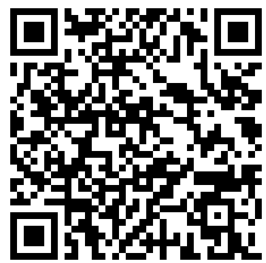

\author{
${ }^{1}$ Dr. Rafael Méndez Sánchez \\ Investigador independiente, Jalisco, México \\ rafamen.dez@hotmail.com \\ https://orcid.org/0000-0003-2143-3115
}

DOI: https://doi.org/10.31434/rms.v3i10.141

\title{
RESUMEN
}

Trastorno motor primario de etiología desconocida con pérdida de la peristalsis esofágica y fallo en la relajación del esfínter esofágico inferior en la deglución. Dando obstrucción funcional y retención de alimentos. Es el desorden funcional más común y que con mayor frecuencia requiere tratamiento quirúrgico. La clínica es gradual e insidiosa, lo más común es la disfagia progresiva, asociada a tos nocturna, pérdida de peso y debilidad o dolor retroesternal urente.

El primer estudio por realizar es el esofagograma con bario, pero la elección continúa siendo la manometría esofágica. Mientras que la endoscopía se reserva para descartar otras patologías o en sospecha de complicaciones.

El tratamiento busca mejorar los síntomas y prevenir sus complicaciones. Mediante propuestas farmacológicas, intervenciones endoscópicas o quirúrgicas, de las cuales la miotomía de Heller continúa siendo el referente.

PALABRAS CLAVES: acalasia, esófago, disfagia, manometría, miotomía.

\section{ABSTRACT}

A primary motor disorder of unknown etiology with loss of esophageal peristalsis and failure to relax the lower esophageal sphincter in swallowing. Giving functional blockage and food retention. It is the most common functional disorder and most often requires surgical treatment. The clinic is gradual and insidious, the most common is progressive dysphagia, associated with nocturnal cough, weight loss and weakness or retrosternal burning pain.

${ }^{1}$ Médico general, graduado de la Universidad de Guadalajara (UdeG), médico investigador independiente, Jalisco, México. Código médico: 273078. rafamen.dez@hotmail.com

The first study to perform is esophagogram with barium, but the choice continues to be esophageal manometry. While endoscopy is reserved to rule out other pathologies or suspected complications. Treatment seeks to improve symptoms and prevent complications.

Through pharmacological proposals, endoscopic or surgical interventions, of which Heller's myotomy remains the benchmark. 
KEY WORDS: achalasia, esophagus, dysphagia, manometry, myotomy.

\section{INTRODUCCIÓN}

La acalasia esofágica es un trastorno motor primario en la que se presenta una pérdida de la peristalsis a nivel del esófago distal asociado a un fallo en la relajación del esfínter esofágico inferior (EEI) en el proceso de deglución. Dando como resultado una obstrucción funcional al vaciamiento esofágico, que lleva a retención de alimentos, provocando daño a la mucosa del órgano.

La etiología se desconoce, pero la teoría más aceptada indica su relación con la disminución de las células ganglionares inhibitorias del plexo mientérico en la pared esofágica y el EEl, con el resultante desbalance de neurotransmisores (aumento de acetilcolina y péptido $P$, con diminución de óxido nítrico y péptido intestinal vasoactivo) generando la afección de su relajación e hipertonía. Existen otras teorías autoinmunes, neurodegenerativas, infecciosas y geneticas.

\section{EPIDEMIOLOGÍA}

Aunque corresponde al desorden funcional más común y conocida del esófago, su presentación es relativamente rara. Pero se trata del trastorno funcional que con mayor frecuencia requiere tratamiento quirurgico.
Se calcula una incidencia anual de aproximadamente 1.8 casos por 100,000 individuos con una prevalencia de 10-12 casos por 100,000 individuos. Afectando por igual a hombres y mujeres, y aunque puede afectar a cualquier grupo etario, su motor incidencia ocurre en la séptima década de la vida y un segundo pico a los 20-40 años.

\section{CLÍNICA}

Presenta una progresión gradual y de inicio insidioso y síntomas inespecíficos, lo que retrasa su diagnóstico, llegando a pasar hasta 24-30 meses antes de iniciar el protocolo de estudio.

La manifestación más común es la disfagia a solidos que progresa a líquidos y posteriormente a la regurgitación de saliva y alimentos blandos, típicamente de sabor "suave", a diferencia de la ERGE que presentan acido gástrico. Característicamente estos síntomas se agravan con el estrés y con líquidos fríos. Otros datos compatibles son la tos nocturna o micro aspiraciones y pérdida de peso involuntaria con debilidad.

En ciertos casos, aparece dolor retroesternal de tipo urente que mejora con la emesis de alimentos no digeridos.

A medida que progresa la anatomía y función esofágica se van modificando, pudiendo generar, hasta en el $10 \%$ de los casos, megaesófago. 


\section{CLASIFICACIÓN}

Primaria o idiopática: En la actualidad se describen 3 subtipos según la manometría de alta resolución:

- Tipo I clásica

- Tipo Il con presurización esofágica

- Tipo III espástica.

Secundaria o pseudoacalasia: Grupo de trastornos que simulan clínica, radiológica y manométricamente a la acalasia idiopática.

- No tumorales: Por ejemplo: enfermedad de Chagas, esofagitis eosinofilica, amiloidosis, etc.

- Tumorales: Adenocarcinoma de cardias, Linfoma, Carcinoma epidermoide de esófago, etc.

\section{DIAGNÓSTICO}

Se establece ante la sospecha por historia clínica y realización de prueba terapéutica fallida de 4 semanas con inhibidores de la bomba de protones (IBP). El primer estudio específico por realizar es el esofagograma con bario en el que se pueden encontrar: dilatación esofágica, estrechamiento de la unión gastroesofágica (signo de "pico de pájaro") y pobre vaciamiento esofágico. La evaluación endoscópica se recomienda para descartar otras patologías que simulen el cuadro o que presenten una complicación de esta.

Sin embargo, para lograr el diagnostico se requiere de manometría esofágica, que demuestra una ausencia de peristalsis esofágica con una pobre relajación, con una sensibilidad de más del $90 \%{ }^{2}$ Hasta en el $50 \%$ se encuentra hipertensión del EEI (mayor a $40 \mathrm{mmHg}$ ). Actualmente la manometría de alta resolución (a diferencia de la convencional) tienen mayor sensibilidad en el diagnóstico, ya que confirma la acalasia y establece la morfología de la unión gastroesofágica.

Sin embargo, encuentra su limitación en la realización, ya que solo pocos centros especializados cuentan con dicha técnica.

\section{TRATAMIENTO}

La lesión nerviosa de esta enfermedad es irreversible, por lo que el tratamiento busca mejorar los síntomas y prevenir sus complicaciones.

Tratamiento médico: Dinitrato de Isosorbida $(5-20 \mathrm{mg})$ vía SL o Nifedipina (10-30 mg) 15 a 30 minutos antes de los alimentos, acatan disminuyendo el tono del EEl. Tienen solo respuestas transitorias, por lo que se prefieren en pacientes mayores o que tienen alto riesgo quirurgico.

Tratamiento endoscópico: Inyección de toxina botulínica (aunque tiene un éxito de $80-85 \%$, puede generar fibrosis de la unión gastroesofágica, dificultando la realización ulterior de la miotomía de Heller), dilatación endoscópica (el más eficaz de este grupo, pero tiene riesgo de perforación y recurrencia), prótesis o miotomía endoscópica peroral (POEM). Se consideran tratamientos a corto plazo y que, en general, deben repetirse.

Tratamiento quirúrgico: Miotomía de Heller más funduplicatura parcial antirreflujo o esofagomiotomía. De elección, sobre todo en jóvenes.

En la actualidad los mejores resultados se obtienen con la dilatación y la cirugía, siendo la miotomía de Heller la que 
produce resultados más duraderos, lo que la coloca como el procedimiento de elección. Con la coca evidencia disponible, la POEM parece ser un procedimiento prometedor, pero solo con estudios a largo plazo que comparen la miotomía de Heller y POEM se podrá determinar el papel de esta nueva técnica en el tratamiento de la acalasia esofágica.

\section{BIBLIOGRAFÍA}

1. Aragón, J. I. (2015). Acalasia: Manifestaciones clínicas y diagnóstico. Revista Médica de Costa Ruca y Centroamérica LXXII, 615, 257-259.

2. Barajas-Fregoso, E. M., Romero-Hernández, T. (2015). Experiencia del manejo quirúrgico de la acalasia esofágica en un hospital de alta especialidad. Revista Médica del Instituto Mexicano del Seguro Social, 53, 84-91.

3. Bayoll, A., Sardiñas, Y. Y., Bayoll, E. H. (2017). Acalasia esofágica: presentación de un caso y revisión de la literatura. IntraMed Journal, 6, 1-6.

4. Jiménez, R. R., Roque, G. R., Anido, E. V. (2015). Estrategias terapéuticas en el tratamiento de la acalasia esofágica. Revista Cubana de Cirugía, 31(4), 318-326.

5. Smith, L. S. (2018). Pacientes con acalasia esofágica: velar por su seguridad. Nursing 2018, $35,59-61$.

Recepción: 10 Setiembre 2018

Aprobación: 25 Setiembre de 2018

\section{(ㅇ) (1) \&}

Reconocimiento-No Comercial 4.0 Internacional (CC BY-NC 4.0)- (BY) Debe reconocer adecuadamente la autoría, proporcionar un enlace a la licencia e indicar si se han realizado cambios<. Puede hacerlo de cualquier manera razonable, pero no de una manera que sugiera que tiene el apoyo del licenciador o lo recibe por el uso que hace. (NC) No puede utilizar el material para una finalidad comercia. 